Pacific Journal of Mathematic 


\title{
PERMANENCE PROPERTIES OF NORMAL STRUCTURE
}

\author{
Thomas LANDES
}

\begin{abstract}
A new characterization of normal structure is given, which allows to prove permanence properties of normal structure such as preservation under finite direct-sum-operations - e.g., the $l_{p}^{N}$-direct sums, $1<p \leq$ $\infty$-as well as under certain infinite direct-sum-operations - e.g., the $l_{p}$-direct sums, $1<p<\infty$.

Furthermore, it is shown that a normed space has isonormal structure - i.e., it is isomorphic to a normally structured space - if and only if it can be mapped by a continuous linear one-to-one operator into some normally structured space.

Finally, some problems are discussed, such as preservation of normal structure under the $l_{1}^{2}$-direct-sum-operation. To solve the latter at least partially, a sum-property is introduced which implies normal structure. This sum-property is implied by all known sufficient conditions for normal structure, and it is preserved under all finite direct-sum-operations.
\end{abstract}

1. Introduction. The aim of this paper is to prove permanence properties of normal structure such as: The $l_{p}^{2}$-direct sum of two Banach spaces $X$ and $Y$-i.e., the direct sum of $X$ and $Y$ endowed with the norm $\|(x, y)\|=\left(\|x\|^{p}+\|y\|^{p}\right)^{1 / p}$ - has normal structure whenever $X$ and $Y$ both do so.

The concept of normal structure has been first introduced by Brodskii and Mil'man [5]:

Definition 1. The subset $A$ of the normed space $X$ is said to have normal structure (or to be normally structured) if every bounded convex nonvoid subset $C$ of $A$ with positive diameter

$$
d:=\operatorname{diam} C:=\sup \{\|x-y\| x, y \in C\}>0
$$

is contained in some ball centered in $C$ with radius smaller than $d$.

In the obvious fashion, the notion of normal structure can be carried over to subsets of a locally convex topological vector space (lctvs, hereafter) by replacing the norm by some continuous seminorm $q$ (see [30], [31], [33]). In this case, we speak of normal structure with respect to $q$. Normal structure with respect to a system $Q$ of continuous seminorms means normal structure with respect to all $q \in Q$. 
Normal structure is a fundamental tool in fixed point theory of nonexpansive mappings. As an example, we mention the celebrated fixed point theorem of Browder [6], Göhde [22] and Kirk [27] (see also [7], [21], [29]):

Every nonexpansive selfmapping of a weakly compact convex normally structured nonvoid subset of a Banach space has a fixed point.

Several approaches to the Browder-Göhde-Kirk-theorem are given in [29]; generalizations may be found, for example, in [2], [3], [18], [30], [32], [38], [39].

The very first examples of normally structured sets are the compact sets and all subsets of uniformly convex spaces. A complete survey of conditions presently known to be sufficient for normal structure is given in [28] (see also the appendix). Spaces lacking normal structure are $c_{0}(I)$, $l_{1}(I), l_{\infty}(I), I$ infinite, $C(0,1), L^{1}, L^{\infty}$, etc. More examples are given in [8].

In view of applications, one naturally is interested in finding the system $Q_{n s}(A)$ of all those continuous seminorms on the lctvs $E$ with respect to which the given subset $A$ of $E$ has normal structure. In [31], the author has proved:

Every continuous seminorm belongs to $Q_{n s}(A)$ if and only if every bounded convex subset of $A$ is precompact.

Generally, it is rather hopeless to determine $Q_{n s}(A)$ even if one considers only equivalent norms of Banach spaces. Establishing permanence properties and finding sufficient conditions for normal structure can be understood as a good approximation of $Q_{n s}(A)$.

2. The notation. In order to make the notation simpler and for the sake of clarity, we introduce the following notation:

(1) Given a product space or a space with a basis, we always denote the $i$ th component of an element $x$ by $x(i)$ and reserve the small letters $i$ and $j$ for this purpose.

(2) In order to avoid confusion with indices, we always use bold face letters such as $\mathbf{x}$ to denote the sequence $\mathbf{x}=\left\{x_{n}\right\}$. Sometimes, we also denote the range of $\mathbf{x}$, i.e. the set $\left\{x_{n} \mid n \in \mathbf{N}\right\}$, by $\mathbf{x}$.

(3) We call a normed space $Z$ a substitution space (with index set $I \neq \varnothing$, where $I$ may have any cardinality) whenever $Z$ has a (Schauder-) basis $\left(e_{i}\right)_{i \in I}$ (unconditional if $I$ is uncountable) and the norm of $Z$ is monotone, i.e., $\|z\| \leq\|\tilde{z}\|$ whenever $0 \leq z(i) \leq \tilde{z}(i)$ for all $i \in I(z, \tilde{z} \in Z)$.

Examples of substitution spaces are:

(a) $l_{p}(I), 1 \leq p<\infty$, or $c_{0}(I)$ for any set $I$, e.g. $l_{p}=l_{p}(\mathbf{N}), c_{0}=c_{0}(\mathbf{N})$.

(b) $\mathbf{R}^{N}$ with any monotone norm, e.g. $l_{p}^{N}=l_{p}(\{1, \ldots, N\}), 1 \leq p \leq \infty$. 
Given a substitution space $Z$ with index set $I$ and given a family $\left(X_{i}\right)_{l \in I}$ of normed spaces, then the subadditive, positively homogeneous operator $S$ mapping all those $x \in \prod_{i \in I} X_{l}$, for which $\sum_{i \in I}\|x(i)\| e_{l}$ is an element of $Z$, onto this sum is called the substitution operator. The $Z$-direct-sum $\left(\Sigma_{l \in I} \oplus X_{l}\right)_{Z}$ of the family $\left(X_{i}\right)$ is defined to be the domain of $S$ endowed with the norm $\|x\|:=\|S x\|_{z}$. We use the projections $P_{J} z=\sum_{j \in J} z(j) e_{j}$ and $\tilde{P}_{J} z=z-P_{J} z, J \subset I, z \in Z$.

(4) Hereafter, $X, Y, Z$ are normed spaces.

(5) Given a substitution space $Z$, a property $P$ defined for normed spaces is said to be preserved under the $Z$-direct-sum-operation, if the $Z$-direct sum of the family $\left(X_{i}\right)$ of normed spaces satisfies $P$ whenever all $X_{l}$ do so.

(6) We denote the convex (closed convex, resp.) hull of $A \subset X$ by $\operatorname{conv} A\left(\overline{\operatorname{conv}} \mathrm{A}\right.$, resp.). We write $\mathbf{x} \subset{ }_{c} A$ if $\operatorname{conv} \mathbf{x}$ is contained in $A$.

(7) The distance of $x \in X$ from $A \subset X$ is denoted by $\operatorname{dist}(x, A)=$ $\inf \{\|x-a\| \mid a \in A\}$.

(8) The mean of $n$ elements $v_{1}, \ldots, v_{n}$ of a vector space is denoted by

$$
\overline{v_{n}}=\frac{1}{n} \sum_{k=1}^{n} v_{k} \text {. }
$$

3. Characterizations of normal structure. In order to check normal structure for a given set one needs suitable equivalent conditions. Therefore, we give a list of those conditions. We only formulate the norm-version, the seminorm-version can be obtained in the obvious way.

Proposition 1. Each of the following conditions is equivalent to the statement that the given subset $A$ of $X$ has normal structure (abbreviated by (NS)).

(NS1) There is no diametral sequence $\mathbf{x} \subset_{c} A$, where $\mathbf{x}$ is called diametral if

$$
0<\lim _{n \rightarrow \infty}\left\|x_{n}-x\right\|=\operatorname{diam} \mathbf{x}<\infty \quad \text { for all } x \in \operatorname{conv} \mathbf{x} .
$$

(NS2) There is no sequence $\mathbf{x} \subset_{c} A$ with

$$
0<\lim _{n \rightarrow \infty}\left\|x_{n}-\overline{x_{k}}\right\|=\operatorname{diam} \mathbf{x}<\infty \quad \text { for all } k \in \mathbf{N} .
$$

(NS3) There is no sequence $\mathbf{x} \subset_{c} A$ with

$$
0<\lim _{n \rightarrow \infty} \operatorname{dist}\left(x_{n+1}, \operatorname{conv}\left\{x_{k} \mid k \leq n\right\}\right)=\operatorname{diam} \mathbf{x}<\infty .
$$


(NS4) There is no bounded non-constant sequence $\mathbf{x} \subset_{c} A$ such that

$$
\left\|x_{n+1}-\bar{x}_{n}\right\| \geq \operatorname{diam} \mathbf{x}-c_{n} \text { for all } n \in \mathbf{N}
$$

for some sequence c of positive reals satisfying $n c_{n} \rightarrow 0$.

(NS5) For every bounded non-constant sequence $\mathbf{x} \subset_{C} A$ there is a $c>0$ such that

$$
\left\|x_{n+1}-\overline{x_{n}}\right\| \leq \operatorname{diam} \mathbf{x}-\frac{c}{n} \quad \text { for all } n \text { bigger than some } m \in \mathbf{N} .
$$

(NS6) There is no bounded non-constant sequence $\mathbf{x} \subset_{c} A$ with

$$
\lim _{n \rightarrow \infty} n\left(\operatorname{diam} \mathbf{x}-\left\|x_{n+1}-\overline{x_{n}}\right\|\right)=0 .
$$

(NS7) There is no sequence $\mathbf{x} \subset_{c} A$ such that $\lim _{n \rightarrow \infty} \Delta \Sigma_{n} \mathbf{x}=0$,

$$
\Delta \Sigma_{n} \mathbf{x}:=\sum_{k=1}^{n}\left\|x_{n+1}-x_{k}\right\|-\left\|\sum_{k=1}^{n}\left(x_{n+1}-x_{k}\right)\right\|
$$

and such that $0<\lim _{n \rightarrow \infty}\left\|x_{n+1}-x_{k}\right\|=\operatorname{diam} \mathbf{x}<\infty$ for all $k \in \mathbf{N}$.

Moreover, if the statement

(NS8, c) For every bounded non-constant sequence $\mathbf{x} \subset_{c} A$ we have

$$
\left\|x_{n+1}-\overline{x_{n}}\right\| \leq \operatorname{diam} \mathbf{x}-c_{n} \text { for some } n \in \mathbf{N} \text {. }
$$

holds true for some sequence $\mathbf{c}$ of positive reals satisfying $n c_{n} \rightarrow 0$, then $A$ has normal structure and, hence, all the properties listed above are satisfied and,

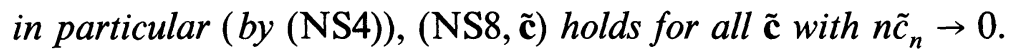

The following diagram illustrates the system of implications which prove Proposition 1:

$$
\begin{aligned}
& (\exists \mathrm{c})(\mathrm{NS} 8, \mathrm{c}) \stackrel{1}{\Leftarrow}\left(\mathrm{NS} 8,\left\{n^{-2}\right\}\right) \stackrel{2}{\Leftrightarrow}(\mathrm{NS} 3) \stackrel{3}{\Leftrightarrow}(\mathrm{NS}) \stackrel{4}{\Leftrightarrow}(\mathrm{NS} 1) \stackrel{5}{\Leftrightarrow}(\mathrm{NS} 7) \\
& 6 \Downarrow \quad 7 \Uparrow \quad 8 \Uparrow \Downarrow 9 \\
& (\mathrm{NS} 5) \underset{10}{\Rightarrow}(\mathrm{NS} 6) \underset{11}{\Rightarrow}(\forall \mathrm{c})(\mathrm{NS} 8, \mathrm{c}) \underset{12}{\Leftrightarrow}(\mathrm{NS} 4)
\end{aligned}
$$

The implications $1,7,8,10,11,12$ are easy to verify and left to the reader. The equivalences 2, 3, 4 are due to Lim [32], [33] and Brodskii-Mil'man [5]. The implications 9 and 5 are consequences of Remark 1.1 and Remark 1.2, respectively (see below). It remains to show 6 .

Proof of 6. If (NS5) does not hold, then there is a bounded sequence $\mathbf{x} \subset \subset_{c} A$ such that $\liminf _{m \rightarrow \infty} m\left(d-\left\|x_{m+1}-\overline{x_{m}}\right\|\right)=0, d:=\operatorname{diam} \mathbf{x}>0$. We fix $\mathbf{c}$ with $n c_{n} \rightarrow 0$. Then, there is an increasing sequence $\{m(n)\}$ in $\mathbf{N}$ 
such that

$$
m(n)\left(d-\left\|x_{m(n)+1}-\overline{x_{m(n)}}\right\|\right) \leq n c_{n} \quad \text { for all } n \in \mathbf{N} .
$$

We set $m(0)=0$ and $u_{n}=x_{m(n-1)+1}$. Observation 1 below implies:

$$
\begin{aligned}
&\left\|u_{n+1}-\overline{u_{n}}\right\|=\left\|x_{m(n)+1}-\frac{1}{n} \sum_{k=0}^{n-1} x_{m(k)+1}\right\| \\
& \geq d-\frac{m(n)}{n}\left(d-\left\|x_{m(n)+1}-\overline{x_{m(n)}}\right\|\right) \geq d-c_{n} \geq \operatorname{diam} \mathbf{u}-c_{n} .
\end{aligned}
$$

So, (NS8, c) cannot hold.

In the proof of Proposition 1 as well as of Remark 1 the following is used:

Observation. (1) Given a sequence $\mathbf{x}$ in $X$ and scalars $\lambda_{1}, \ldots, \lambda_{n} \geq 0$, $\sum_{k=1}^{n} \lambda_{k}=1$, we have $\left(\lambda:=\max _{k \leq n} \lambda_{k}\right)$ :

$$
\begin{aligned}
& \left\|x_{n+1}-\sum_{k=1}^{n} \lambda_{k} x_{k}\right\| \geq \lambda n\left\|x_{n+1}-\overline{x_{n}}\right\|-\sum_{k=1}^{n}\left(\lambda-\lambda_{k}\right)\left\|x_{n+1}-x_{k}\right\| \\
& =\sum_{k=1}^{n} \lambda_{k}\left\|x_{n+1}-x_{k}\right\|-\lambda \Delta \Sigma_{n} \mathbf{x} \geq \lambda n\left\|x_{n+1}-\overline{x_{n}}\right\|-(\lambda n-1) \operatorname{diam} \mathbf{x} \\
& =\operatorname{diam} \mathbf{x}-\lambda n\left(\operatorname{diam} \mathbf{x}-\left\|x_{n+1}-\bar{x}_{n}\right\|\right) .
\end{aligned}
$$

(2) Given a bounded sequence $\mathbf{x}$ in $X$ there can be constructed a subsequence $\mathbf{u}$ of $\mathbf{x}$ such that, for all $k \in \mathbf{N}, a_{k}=\lim _{n \rightarrow \infty}\left\|u_{n}-u_{k}\right\|$ exists and

$$
||\left|u_{n+1}-u_{k} \|-a_{k}\right| \leq n^{-2} \text { for all } n \geq k .
$$

If, additionally, $\mathbf{x}$ is limit-affine, then $\mathbf{u}$ can be chosen so that, additionally,

$$
||\left|u_{n+1}-\bar{u}_{n}\right|\left|-\overline{a_{n}}\right| \leq n^{-2} \text { for all } n \in \mathbf{N}
$$

Definition 2. A sequence $\mathbf{x}$ in $X$ is called limit-affine if $\Lambda(x):=\lim _{n \rightarrow \infty}\left\|x_{n}-x\right\|$ exists for every $x \in \operatorname{conv} \mathbf{x}$ and $\Lambda$ is affine on conv $\mathbf{x}$.

Every diametral sequence is limit-affine, it is even limit-constant, i.e., it is limit-affine with the additional property that the above mapping $\Lambda$ is positive and constant on conv $\mathbf{x}$. 
REMARK 1. (1) Let $\mathbf{x}$ be a sequence in $X$ such that

(i) $a_{k}:=\lim _{n \rightarrow \infty}\left\|\underline{x_{n}}-x_{k}\right\|$ exists for all $k \in \mathbf{N}$.

Then $\liminf _{m \rightarrow \infty}\left\|x_{m}-\overline{x_{n}}\right\|<\overline{a_{n}}$ whenever there is some $x=\Sigma \lambda_{k} x_{k} \in$ $\operatorname{conv}\left\{x_{k} \mid k \leq n\right\}$ for which $\liminf _{m \rightarrow \infty}\left\|x_{m}-x\right\|<\sum \lambda_{k} a_{k}$. Thus, $\mathbf{x}$ is limit-affine if and only if

(ii) $\lim _{m \rightarrow \infty}\left\|x_{m}-\overline{x_{n}}\right\|=\overline{a_{n}}$ for all $n \in \mathbf{N}$.

Moreover, if $\mathbf{x}$ is limit-affine, then so is every subsequence of $\mathbf{x}$ and

(iii) $\lim _{n \rightarrow \infty}\left\|x_{n}-x\right\|=\lim _{n \rightarrow \infty} a_{n}$ whenever $x$ is a weak cluster-point of $\mathbf{x}$ and if the limit on the right hand side exists.

(2) If $\mathbf{x}$ is limit-affine, then there is a subsequence $\mathbf{u}$ of $\mathbf{x}$ such that

(iv) $\lim _{n \rightarrow \infty} \Delta \Sigma_{n} \mathbf{u}=0$.

Vice versa, if $\mathbf{x}$ is any sequence with (i) and (iv), then $\mathbf{x}$ is limit-affine. Thus, if $\mathbf{x}$ satisfies (iv), then it has a limit-affine subsequence.

(3) If $\mathbf{x}$ is limit-affine, then it has a subsequence $\mathbf{v}$ such that

(v) $\lim _{m \rightarrow \infty}\left\{\sum_{k=1}^{m} \lim _{n \rightarrow \infty}\left\|v_{n}-v_{k}\right\|-\left\|\sum_{k=1}^{m}\left(v_{m+1}-v_{k}\right)\right\|\right\}=0$.

(4) Every bounded sequence $\mathbf{x}$ has a subsequence $\mathbf{v}$ such that $\liminf _{n \rightarrow \infty} \Delta \Sigma_{n} \mathbf{x} \geq \lim \sup _{n \rightarrow \infty} \Delta \Sigma_{n} \mathbf{u}$ for every subsequence $\mathbf{u}$ of $\mathbf{v}$.

Proof. (1) By observation 1 we obtain for $x=\sum_{k=1}^{n} \lambda_{k} x_{k}$ and $\lambda=$ $\max _{k \leq n} \lambda_{k}$ :

$$
\begin{aligned}
\liminf _{m \rightarrow \infty}\left\|x_{m}-\overline{x_{n}}\right\| & \leq \frac{1}{\lambda n} \liminf _{m \rightarrow \infty}\left(\left\|x_{m}-x\right\|+\sum_{k=1}^{n}\left(\lambda-\lambda_{k}\right)\left\|x_{m}-x_{k}\right\|\right) \\
& =\overline{a_{n}}+\frac{1}{\lambda n}\left(\liminf _{m \rightarrow \infty}\left\|x_{m}-x\right\|-\sum_{k=1}^{n} \lambda_{k} a_{k}\right) .
\end{aligned}
$$

If $x$ is a weak cluster-point of $\mathbf{x}$, then $x \in \cap_{n \in \mathbf{N}} \overline{\operatorname{conv}}\left\{x_{k} \mid k \geq n\right\}$. If $\mathbf{x}$ is limit-affine and $\lim _{n \rightarrow \infty}\left\|x_{n}-x_{k}\right\|=a_{k} \rightarrow a$ as $k \rightarrow \infty$, then

$$
\lim _{n \rightarrow \infty}\left\|x_{n}-x\right\| \in \bigcap_{n \in \mathbf{N}} \overline{\operatorname{conv}}\left\{a_{k} \mid k \geq n\right\}=\{a\} .
$$

(2) Let $\mathbf{x}$ be limit-affine. Choose $\mathbf{u}$ according to observation 2 . Then

$$
0 \leq \Delta \Sigma_{n} \mathbf{u}=\sum_{k=1}^{n}\left\|u_{n+1}-u_{k}\right\|-n\left\|u_{n+1}-\overline{u_{n}}\right\| \leq \sum_{k=1}^{n} a_{k}-n \overline{a_{n}}+\frac{2}{n}=\frac{2}{n} .
$$

Vice versa, let $\mathbf{x}$ satisfy (i) and (iv). By observation 1 , letting $m \rightarrow \infty$, we get:

$$
\overline{a_{n}} \leftarrow \frac{1}{n} \sum_{k=1}^{n}\left\|x_{m}-x_{k}\right\| \geq\left\|x_{m}-\overline{x_{n}}\right\| \geq \frac{1}{n} \sum_{k=1}^{n}\left\|x_{m}-x_{k}\right\|-\frac{1}{n} \Delta \Sigma_{m-1} \mathbf{x} \rightarrow \overline{a_{n}} .
$$


Now, consider $v_{n}=x_{m(n)}$, where $m(n)$ is increasing. Set $m=m(n+1)$. By observation 1, we obtain:

$$
\begin{aligned}
\Delta \Sigma_{n} \mathbf{v} & =\sum_{k=1}^{n}\left\|v_{n+1}-v_{k}\right\|-n\left\|x_{m}-\sum_{k=1}^{n} \frac{1}{n} x_{m(k)}\right\| \\
& \leq \sum_{k=1}^{n}\left\|v_{n+1}-v_{k}\right\|-n\left(\sum_{k=1}^{n} \frac{1}{n}\left\|x_{m}-x_{m(k)}\right\|-\frac{1}{n} \Delta \Sigma_{m-1} \mathbf{x}\right) \\
& =\Delta \Sigma_{m(n+1)-1} \mathbf{x} .
\end{aligned}
$$

This proves the last assertion of 2 .

(3) This can be shown exactly like the first assertion of 2 .

(4) Choose $m(n)$ so that $L=\liminf _{n \rightarrow \infty} \Delta \Sigma_{n} \mathbf{x}=\lim _{n \rightarrow \infty} \Delta \Sigma_{m(n)} \mathbf{x}$. Set $u_{n}=x_{m(n)+1}$. Then, if $p(n)$ is increasing, we obtain as in the last step of 2 for $v_{n}=u_{p(n)}$ :

$$
\begin{aligned}
\limsup _{n \rightarrow \infty} \Delta \Sigma_{n} \mathbf{v} & \leq \limsup _{n \rightarrow \infty} \Delta \Sigma_{p(n+1)-1} \mathbf{u} \\
& \leq \limsup _{n \rightarrow \infty} \Delta \Sigma_{m(p(n+1))} \mathbf{x}=L .
\end{aligned}
$$

Remark 1 is a good tool for proving the next Theorem 1 and will also be used later on (e.g. \$7).

Statement (NS1) excludes the existence of a limit-constant sequence such that, in addition, the corresponding constant is the diameter of the sequence. We show that this additional condition can be dropped.

THEOREM 1. Given a subset $A$ of $X$, the following are equivalent. (NS) $A$ has normal structure.

(NS1)* There is no limit-constant sequence with convex hull in $A$.

(NS2)* There is no bounded sequence $\mathbf{x} \subset_{c} A$ such that

$$
\lim _{n \rightarrow \infty}\left\|x_{n}-x_{k}\right\|=\lim _{n \rightarrow \infty}\left\|x_{n}-\bar{x}_{l}\right\|>0 \quad \text { for all } k, l \in \mathbf{N} .
$$

(NS7)* There is no sequence $\mathbf{x} \subset_{c} A$ such that $\lim _{n \rightarrow \infty} \Delta \Sigma_{n} \mathbf{x}=0$ and

$$
\lim _{n \rightarrow \infty}\left\|x_{n}-x_{k}\right\|=\lim _{n \rightarrow \infty}\left\|x_{n}-x_{l}\right\|>0 \quad \text { for all } k, l \in \mathbf{N} \text {. }
$$

Proof. In view of Remark 1, we only have to show:

(NS2) $\Rightarrow(\mathrm{NS} 1)^{*}$. Let $\mathbf{x} \subset_{c} A$ be limit-constant with corresponding constant $a>0$. We choose a subsequence $\mathbf{v}$ of $\mathbf{x}$ such that $\left\|v_{n+1}-v_{k}\right\| \leq$ $a\left(1+\varepsilon_{n}\right), \varepsilon_{n}:=1 / n$, for all $n \geq k$. Setting $\alpha_{n}=\left(1+\varepsilon_{n}\right)^{-1}, \beta_{n}=1-\alpha_{n}$ $=\varepsilon_{n} \alpha_{n}$ and $u_{n}=\alpha_{n} v_{n+1}+\beta_{n} v_{1} \in$ conv $\mathbf{x}$, we obtain: 


$$
\begin{aligned}
\left\|u_{n}-u_{k}\right\| & =\left\|\alpha_{n} v_{n+1}-\alpha_{k} v_{k+1}-\left(\beta_{k}-\beta_{n}\right) v_{1}\right\| \\
& \leq\left(\alpha_{k}+\beta_{k}-\beta_{n}\right) a\left(1+\varepsilon_{n}\right)=a,
\end{aligned}
$$

and

$$
\left\|u_{n}-\overline{u_{k}}\right\|=\left\|v_{n+1}-\frac{1}{k} \sum_{\nu=1}^{k} \alpha_{\nu} v_{\nu+1}-\bar{\beta}_{k} v_{1}+\beta_{n}\left(v_{1}-v_{n+1}\right)\right\| \rightarrow a
$$

as $n \rightarrow \infty$.

Therefore, $a=\operatorname{diam} \mathbf{u}$ and $\operatorname{conv} \mathbf{u} \subset \operatorname{conv} \mathbf{x} \subset A$ which contradicts (NS2).

In view of fixed point theorems it is desirable to find conditions under which every relatively weakly compact subset of a given normed space has normal structure. Such a space is said to have weakly normal structure. Of course, all Schur spaces (weak compactness coincides with strong compactness) have weakly normal structure. Since every $l_{1}(I)$-direct sum of finite dimensional spaces ("Schur spaces" suffices) is Schur, those spaces have weakly normal structure. This is in contrast to a false remark in [31, Remark 3]. In particular, $l_{1}$ has weakly normal structure but not normal structure.

By restricting each of the statements (NS $\nu), \nu \leq 7,(\mathrm{NS} 8, \mathrm{c}),(\mathrm{NS} \nu)^{*}$, $\nu=1,2,7$, to sequences which converge weakly to 0 , we obtain characterizations for weakly normal structure. Moreover, looking at Remark 1, we have:

Proposition 2. A normed space $X$ has weakly normal structure if and only if there is no sequence $\mathbf{x}$ in $X$ such that $\mathbf{x}$ converges weakly to 0 ,

$$
1=\lim _{n \rightarrow \infty}\left\|x_{n}-x_{k}\right\|=\lim _{n \rightarrow \infty}\left\|x_{n}\right\| \quad \text { for all } k \in \mathbf{N}, \text { and } \lim _{n \rightarrow \infty} \Delta \Sigma_{n} \mathbf{x}=0 .
$$

4. Finite direct sums. The first permanence result for normal structure is due to Belluce, Kirk and Steiner [4]:

Proposition 3. The $l_{\infty}^{N}$-direct sum of $N$ normally structured normed spaces again has normal structure.

Of course, we can replace norms by seminorms. So, we obtain:

COROLlaRY 1. Let the lctvs $E$ have normal structure with respect to the system $Q$ of continuous seminorms. Then, $E$ has normal structure also with 
respect to the saturation of $Q$, that is the following system of seminorms:

$$
V Q:=\left\{\max _{\nu \leq N} \alpha_{\nu} q_{\nu} \mid \alpha_{\nu}>0, q_{\nu} \in Q, \nu \leq N \in \mathbf{N}\right\}
$$

COROLlaRY 2. Let $\left(E_{i}\right)_{i \in I}$ be a family of lctvs each of them having normal structure with respect to a seminorm-system $Q_{i}$ which defines the topology of $E_{i}$. Let the topology of the locally convex product $E=\prod_{i \in I} E_{i}$ be induced by the canonical system $Q^{\infty}=\left\{\max _{j \in J} q_{j} \mid q_{j} \in V Q_{j}, J\right.$ finite $\left.\subset I\right\}$, $\left(\max _{j \in J} q_{j}\right)(x):=\max _{j \in J} q_{j}(x(j))$. Then, $E$ has normal structure with respect to $Q^{\infty}$.

Using our new characterization, we can improve Proposition 3. The condition $(*)$ used in the next theorem is satisfied in particular, if $Z$ is strictly convex, but also for $Z=l_{\infty}^{N}$, yet not for $Z=l_{1}^{N}$.

THEOREM 2. Let $Z$ be a substitution space with index set $I=\{1, \ldots, N\}$ such that

(*) $\|z+\tilde{z}\|<2$ whenever $\|z\|=\|\tilde{z}\|=1, z(i) \geq 0, \tilde{z}(i) \geq 0$ for all $i \in I$, and $z(i)=\tilde{z}(i)$ only for those $i \in I$ for which $z(i)=\tilde{z}(i)=0$. Then, normal structure is preserved under the Z-direct-sum-operation.

Proof. Assume that $\mathbf{x}$ is diametral with diameter $d$. Passing to subsequences we may assume that:

(i) $z_{k}(i):=\lim _{n \rightarrow \infty}\left\|x_{n}(i)-x_{k}(i)\right\|$ exists for all $k \in \mathbf{N}$ and $i \in I$.

(ii) $\left\|z_{k}\right\|=d$ for all $k \in \mathbf{N}, z_{k}=\left(z_{k}(i)\right)=\lim _{n \rightarrow \infty} S\left(x_{n}-x_{k}\right)$.

(iii) $d_{n}:=n\left(d-\left\|x_{n+1}-\overline{x_{n}}\right\|\right) \rightarrow 0$ as $n \rightarrow \infty$.

(iv) Given $i \in I, \mathbf{z}(i)$ is either increasing, decreasing or constant. We may clearly drop those components $i$ for which $\mathrm{z}(i) \equiv 0$. Let $J$ be the set of those $j \in I$ for which $\mathbf{z}(j) \equiv z(j)$ is constant. Using (NS7)* for $X_{j}$ (and Remark 1), we find an $\varepsilon>0$ and an $m \in \mathbf{N}$ such that

$$
\begin{aligned}
& \left\|\sum_{\nu=1}^{n}\left(x_{n+1}(j)-x_{\nu}(j)\right)\right\| \\
& \quad \leq \sum_{\nu=1}^{n}\left\|x_{n+1}(j)-x_{\nu}(j)\right\|-\varepsilon \quad \text { for all } n \geq m \text { and } j \in J
\end{aligned}
$$

For $i \in I \backslash J$ we have

$$
\left\|\sum_{\nu=1}^{n}\left(x_{n+1}(i)-x_{\nu}(i)\right)\right\| \leq \sum_{\nu=1}^{n}\left\|x_{n+1}(i)-x_{\nu}(i)\right\| .
$$


Hence, using the monotony of the norm of $Z$ and the definition of $d_{n}$, we obtain:

$$
\begin{aligned}
& n d-d_{n}=\left\|\sum_{\nu=1}^{n}\left(x_{n+1}-x_{\nu}\right)\right\| \leq\left\|\sum_{\nu=1}^{n} S\left(x_{n+1}-x_{\nu}\right)-\varepsilon \sum_{j \in J} e_{j}\right\| \\
& \leq\left\|\sum_{k \in L} S\left(x_{n+1}-x_{k}\right)-\varepsilon \sum_{j \in J} e_{j}\right\|+(n-l) d, \\
& n \geq m, L \subset \mathbf{N},|L|=l .
\end{aligned}
$$

Subtracting $(n-l) d$ and passing to the limit for $n \rightarrow \infty$, we obtain:

$$
\left\|\sum_{k \in L} z_{k}-\varepsilon \sum_{j \in J} e_{j}\right\|=l d=\left\|\sum_{k \in L} z_{k}\right\|, \quad l \subset \mathbf{N},|L|=l .
$$

Using the monotony of the norm of $Z$, we conclude that

(v) $\left\|\sum_{k \in L} \tilde{P}_{J} z_{k}\right\|=l d, \quad l \subset \mathbf{N},|L|=l$.

Finally, (v) together with (iv) yields a contradiction to $(*)$.

COROLlaRY 3. Normal structure is preserved under any finite directsum-operation with strictly convex substitution space.

COROLlaRY 4. Normal structure is preserved under the $l_{p}^{N}$-direct-sumoperations for any $p$ with $1<p \leq \infty$.

Corollary 5. If $q_{1}, \ldots, q_{N}$ all belong to $Q_{n s}(A)$ and if \|\| is a monotone norm on $\mathbf{R}^{N}$ satisfying $(*)$, then the continuous seminorm $q$ defined by $q(x):=\left\|\left(q_{i}(x)\right)_{\imath \leq N}\right\|$ belongs to $Q_{n s}(A)$, too.

5. Infinite direct sums. Since one crucial step in the proof of Theorem 2, namely that $\left\|x_{n}-x_{k}\right\| \rightarrow a$ and $\left\|x_{n}(i)-x_{k}(i)\right\| \rightarrow z_{k}(i)$ for all $i \in I$ together imply that $\left\|z_{k}\right\|=a$, works no longer for infinite index sets, we cannot expect that Theorem 2 holds in full generality for infinite index sets, too. But, observing that finite dimensional strictly convex spaces are uniformly convex, the following theorem is seen to generalize Corollary 3 .

THEOREM 3. Normal structure is preserved under any direct-sum-operation with a uniformly convex substitution space.

Proof. Suppose that $\mathbf{x}$ is limit-constant with corresponding constant $a>0$. We may assume that $z_{k}(i):=\lim _{n \rightarrow \infty}\left\|x_{n}(i)-x_{k}(i)\right\|$ exists for all $k \in \mathbf{N}$ and $i \in I$. It suffices to show that $\lim _{n \rightarrow \infty}\left\|x_{n}(i)-\bar{x}_{l}(i)\right\|=z_{k}(i)$ 
for all $k, l \in \mathbf{N}$ and $i \in I$, since this together with (NS2)* for $X_{i}$ implies that, for all $i \in I, \mathbf{z}(i) \equiv 0$, and, hence, that $\mathbf{x}(i)$ is constant contradicting $a>0$.

We have $\left\|S\left(x_{n}-x_{k}\right)\right\|=\left\|x_{\underline{n}}-x_{k}\right\| \rightarrow a,\left\|S\left(x_{n}-\bar{x}_{l}\right)\right\|=\left\|x_{n}-\overline{x_{l}}\right\| \rightarrow$ $a$ and $\left\|\frac{1}{2} S\left(x_{n}-x_{k}\right)+\frac{1}{2} S\left(x_{n}-\bar{x}_{l}\right)\right\| \geq\left\|x_{n}-\frac{1}{2} x_{k}-\frac{1}{2} \bar{x}_{l}\right\| \rightarrow a$, as $n \rightarrow \infty$. From uniform convexity we obtain $S\left(x_{n}-x_{k}\right)-S\left(x_{n}-\overline{x_{l}}\right) \rightarrow 0$ and, hence, $\left\|x_{n}(i)-x_{k}(i)\right\|-\left\|x_{n}(i)-\overline{x_{l}(i)}\right\| \rightarrow 0$ for all $i$.

COROllaRY 6. Normal structure is preserved under the $l_{p}$-direct-sumoperations for all $p$ with $1<p<\infty$.

A short look at the proof of Theorem 3 also shows:

Corollary 7. If the family $\left(q_{i}\right)_{i \in I}$ in $Q_{n s}(A)$ is equicontinuous, i.e., $q_{i} \leq \tilde{q}$ for all $i \in I$ and some continuous seminorm $\tilde{q}$, then, given any $\xi \in l_{p}(I), 1<p<\infty$, the continuous seminorm $q$ defined by

$$
q(x):=\left(\sum_{i \in I}\left|\xi(i) q_{i}(x)\right|^{p}\right)^{1 / p}
$$

belongs to $Q_{n s}(A)$, too.

Furthermore, the hypothesis " $q_{i} \in Q_{n s}(A)$ for all $i \in I$ " can be replaced by the condition that, for some $j \in I, q_{j}$ is a norm belonging to $Q_{n s}(A)$ and $\xi(j) \neq 0$.

The latter result will be used later on $(§ 6)$.

The following example shows that one can (by application of Corollary 7) go round the condition of uniform convexity:

EXAMPLE. Let $Z$ be the space $c_{0}$ with the usual unit vector basis $\left(e_{i}\right)_{l \in \mathbf{N}}$ endowed with the norm $\|z\|:=\left(\|z\|^{2}+\sum_{i=1}^{\infty} 4^{-i}|z(i)|^{2}\right)^{1 / 2}$. Then, $Z$ is not uniformly convex, $Z$ is isomorphic to $c_{0}$, and normal structure is preserved under the $Z$-direct-sum-operation. The latter follows by application of Corollary 7 first for $I=\mathbf{N}, p=2, q_{i}(x)=\|x(i)\|$, and $\xi=\left(2^{-i}\right)$, and then for $I=\{1,2\}, \quad p=2, \quad q_{1}(x)=\sup _{i \in \mathbf{N}}\|x(i)\|, \quad q_{2}(x)=$ $\left(\sum_{i=1}^{n} 4^{-i}|x(i)|^{2}\right)^{1 / 2}, \xi=(1,1)$ and $j=2$.

Thus the $c_{0}$-direct sum of normally structured Banach spaces has isonormal structure, i.e., it can be renormed so that it has normal structure. 
Another condition, a slight generalization of a condition introduced by Gossez and Lami Dozo [23], is also suitable for proving normal structure of direct sums:

Definition 3. Let $Z$ be a normed space with basis $\left(e_{i}\right)_{i \in I}$ (unconditional if $I$ is uncountable). The basis is said to satisfy the $G L D$-condition, if there is a "final" (i.e., $\tilde{J}$ finite $\subset I \Rightarrow \tilde{J} \subset J$ for some $J \in \mathcal{G}$ ) set $\mathcal{g}$ of finite subsets of $I$ with the property that there is a $c<1$ and some $r>1$ such that

$\|z\| \geq r \quad$ whenever $\left\|P_{J} z\right\|=1$ and $\left\|\tilde{P}_{J} z\right\| \geq c \quad$ for some $J \in \mathscr{g}$.

Gossez and Lami Dozo showed in [23] that a Banach space has weakly normal structure whenever it has a basis $\left(e_{n}\right)_{n \in \mathbf{N}}$ satisfying the above condition for all $c>0$ instead of for one $c<1$ (see also [9]).

Proposition 4. Let the basis of the substitution space $Z$ satisfy the GLD-condition. Then, the Z-direct sum of a family of Schur spaces has weakly normal structure.

Proof. If $\mathbf{x}$ is diametral with $\lim _{n \rightarrow \infty}\left\|x_{n}\right\|=\operatorname{diam} \mathbf{x}$ and $x_{n} \rightarrow 0$ weakly, then, since all $X_{i}$ are Schur, $\left\|x_{n}(i)\right\| \rightarrow 0$ for all $i \in I$ as $n \rightarrow \infty$. Thus, a contradiction to the GLD-condition can be obtained in exactly the same way as for $X_{i}=\mathbf{R}$ (see [23] or [4]).

6. Isonormal structure. The normed space $X$ is said to have isonormal structure if it is isomorphic to a normally structured space, i.e. if $Q_{n s}(X)$ contains an equivalent norm.

If every space isomorphic to $X$ contains an isometric copy of $l_{\infty}$ (or merely $c_{0}$ ), then $X$ clearly does not have isonormal structure. Examples of such spaces are $l_{\infty}(I)$ for any uncountable $I$ (Partington [35]), $m_{\kappa}(I)=$ $\left\{x \in l_{\infty}(I) \mid \operatorname{card}(\operatorname{supp} x) \leq \kappa\right\}, \boldsymbol{\aleph}_{0} \leq \kappa<\operatorname{card}(I)$ (Partington [35]) and $l_{\infty} / c_{0}$ (Partington [36]).

On the other hand, all superreflexive spaces do have isonormal structure because they are uniformly convexifiable (see [17]).

Zizler [41] has shown that, whenever $X$ can be mapped by a continuous linear one-to-one operator into some space whose norm is uniformly convex in every direction (see [13]), then $X$ can be given an equivalent norm with the same property. Thus ([19], [41]), all such spaces have isonormal structure. To this class belong, for example:

(1) All separable spaces, for example $c_{0}$. 
(2) The space $l_{\infty}$.

(3) The spaces $l_{p}(I)$ for any index set $I$ and any $p$ with $1 \leq p<\infty$.

(4) The space $C B(H)$ of all continuous bounded real valued functions on the completely regular $H$, whenever $H$ admits a $\sigma$-finite Baire measure $\mu$ such that $\mu(A)>0$ if $A$ has nonempty interior $(C B(H)$ is endowed with the topology of uniform convergence).

Using our permanence result, we obtain:

THEOREM 4. A normed space $X$ has isonormal structure if (and only if) there is a continuous linear one-to-one mapping $T$ from $X$ into some normally structured space.

Proof. Apply Corollary 7 for $I=\{1,2\}, q_{1}=\|\cdot\|, q_{2}=\|T \cdot\|, \xi=$ $(1,1), p=2, j=2$.

Especially, we are interested in the space $c_{0}(I)$, since every weakly compactly generated Banach space can be mapped by a continuous linear one-to-one operator into $c_{0}(I)$ for some set $I$ (see [15]). Theorem 4 implies:

Consequence 1. If $c_{0}(I)$ always has isonormal structure, then so does every weakly compactly generated Banach space.

For any positive measure $\mu, L^{1}(\mu)$ can be written as an $l_{1}(I)$-direct sum of a family of $L^{1}\left(\varphi_{i}\right)$-spaces with finite measures $\varphi_{i}$. Since the latter are weakly compactly generated and the formal identity from the $l_{1}(I)$-direct sum into the corresponding $l_{2}(I)$-direct sum is continuous (see also [12]), we obtain:

Consequence 2. If $c_{0}(I)$ always has isonormal structure, then so does $L^{1}(\mu)$ for every positive measure $\mu$.

The behaviour of $c_{0}(I)$ is quite different from that of $l_{\infty}(I)$, since $c_{0}(I)$ always can be endowed with a locally uniformly convex, hence strictly convex, norm, namely Day's norm (see [11], [15], [37]).

Unfortunately, even $c_{0}(\mathbf{N})$ does not have normal structure with respect to Day's norm. In fact, it even does not have weakly normal structure (see [24]). 
7. Open problems. From the preceding section, the following two problems remain open:

Problem 1. Does $c_{0}(I)$ have isonormal structure in case that $I$ is uncountable?

\section{Problem 2. Does $L^{1}(\mu)$ have isonormal structure for every $\mu$ ?}

One naturally is interested in a Corollary 2 like result for the locally convex direct sum $E=\bigoplus_{i \in I} E_{i}$ with its canonical system $Q^{1}=\left\{\sum_{l \in I} q_{i} \mid q_{i}\right.$ $\left.\in V Q_{i}\right\}$ related to systems $Q_{i}$ which define the topology of $E_{i}$, where in $\left(\sum_{i \in I} q_{i}\right)(x):=\sum_{i \in I} q_{i}(x(i))$ the sum on the right hand side ranges over only finitely many non-zero summands.

We observe that every bounded subset $B$ of $E$ lies in some finite step, i.e., there is a finite subset $J$ of $I$ such that $x(i)=0$ whenever $x \in B$ and $i \in I \backslash J$. Thus, to prove that $E$ has normal structure with respect to $Q^{1}$ provided each $E_{i}$ has normal structure with respect to $Q_{i}$, it suffices to give an affirmative answer to the following:

Problem 3. Is normal structure preserved under the $l_{1}^{2}$-direct-sum-operation?

If $X$ and $Y$ both have normal structure but if $\tilde{X}=(X \oplus Y)_{l_{1}^{2}}$ does not, then there is a diametral sequence $\mathbf{u}=(\mathbf{v}, \mathbf{w})$ in $\tilde{X}$ such that $\mathbf{v}$ and $\mathbf{w}$ both are limit-affine and $\left\{\lim _{m \rightarrow \infty}\left\|v_{m}-v_{n}\right\|\right\}_{n}$ is decreasing and $\left\{\lim _{m \rightarrow \infty}\left\|w_{m}-w_{n}\right\|\right\}_{n}$ is increasing (or vice versa). So, we require both $X$ and $Y$ to have the property

(SP) There exists no growing (i.e., $0<\lim _{m \rightarrow \infty}\left\|x_{m}-x_{n}\right\| \leq$ $\lim _{m \rightarrow \infty}\left\|x_{m}-x_{n+1}\right\|$ for all $n \in \mathbf{N}$ ) limit-affine sequence $\mathbf{x}$.

Then $\tilde{X}$ has normal structure. Moreover, it can easily be seen that, in this case, $\tilde{X}$ even satisfies the property (SP) (sum-property, hereafter), too.

Clearly, the sum-property implies normal structure. Using Remark 1, we easily deduce that the sum-property is equivalent to

(SP1) There is no sequence $\mathbf{x}$ such that, for all $n \in \mathbf{N}$,

$$
0<a_{n}:=\lim _{m \rightarrow \infty}\left\|x_{m}-x_{n}\right\| \leq a_{n+1} \text { and } \lim _{m \rightarrow \infty}\left\|x_{m}-\overline{x_{n}}\right\|=\overline{a_{n}} \text {. }
$$

Similarly as in the case of weakly normal structure, $X$ is said to have the weak sum-property if (SP) holds when restricted to weakly convergent sequences. 
We have seen that, for an affirmative solution of Problem 3, it is sufficient to establish the following:

Conjecture. Normal structures implies the sum-property.

This conjecture is supported by the fact that all the conditions known to be sufficient for (weakly) normal structure listed in [28] also imply the (weak) sum-property. Sketches of the proofs of these implications may be found in the appendix.

Finally, we remark that the sum-property is preserved under any finite direct-sum-operation, so that we have:

Consequence 3. If normal structure implies the sum-property, then normal structure is preserved under any finite direct-sum-operation.

Proof. We fix a substitution space $Z$ with finite index set $I$ and show that the sum-property is preserved under the $Z$-direct-sum-operation.

Assume that $\mathbf{x}$ is a growing limit-affine sequence in $X=\left(\sum_{i \in I} \oplus X_{i}\right)_{Z}$. We choose a subsequence $\mathbf{u}$ of $\mathbf{x}$ such that:

(i) $z_{n}(i):=\lim _{m \rightarrow \infty}\left\|u_{m}(i)-u_{n}(i)\right\|$ exists for all $n \in \mathbf{N}$ and $i \in I$.

(ii) $\left\|z_{n}\right\|=a_{n}:=\lim _{m \rightarrow \infty}\left\|u_{m}-u_{n}\right\|$ for all $n \in \mathbf{N}$.

(iii) $0<z_{n}(j) \leq z_{n+1}(j)$ for all $n \in \mathbf{N}$ and all elements $j$ of some $J \subset I$.

(iv) $z_{n}(i)>z_{n+1}(i)$ for all $n \in \mathbf{N}$ and $i \in I \backslash J$.

(v) $\zeta_{n}(i):=\lim _{m \rightarrow \infty}\left\|u_{m}(i)-\overline{u_{n}(i)}\right\|$ exists for all $n \in \mathbf{N}$ and $i \in I$. Observe that we have dropped all components $i$ for which $\mathbf{x}(i)$ is constant. From $a_{n} \leq a_{n+1}$, we know that $J \neq \varnothing$. Using Remark 1.1 and (SP1), we find a $k \in \mathbf{N}$ such that $\zeta_{n}(j)<\overline{z_{n}(j)}$ for all $n \geq k$ and $j \in J$.

Setting $\tilde{z}_{n}:=P_{J} \zeta_{n}+\tilde{P}_{J} \bar{z}_{n}$, we obtain $\overline{a_{n}}=\lim _{m \rightarrow \infty}\left\|u_{m}-\overline{u_{n}}\right\| \leq\left\|\tilde{z}_{n}\right\|$ $\leq\left\|\overline{z_{n}}\right\| \leq \overline{a_{n}}$.

Using the monotony of the norm of $Z$, we obtain $\overline{a_{n}}=\left\|\tilde{z}_{n}\right\|=\left\|\overline{z_{n}}\right\|=$ $\left\|\tilde{P}_{J} \overline{z_{n}}\right\|>\left\|\tilde{P}_{J} \overline{z_{n+1}}\right\|=\overline{a_{n+1}}$ which contradicts $a_{m} \leq a_{m+1}$ for all $m$.

Consequence 3 again gives a motivation to check whether our conjecture is true or not.

8. Appendix. In the appendix, we want to show that all sufficient conditions for (weakly) normal structure listed in [28] are sufficient for the (weak) sum-property, too. The following proofs all are indirect using (SP) or the fact that, if $X$ does not have the weak sum-property (WSP), then 
there is a growing limit-affine sequence $\mathbf{x}$ with $x_{n} \rightarrow 0$ weakly and $\lim _{n \rightarrow \infty} \lim _{m \rightarrow \infty}\left\|x_{m}-x_{n}\right\|=1=\lim _{n \rightarrow \infty}\left\|x_{n}\right\|$.

(1) Uniformly normal structure (see [20]) $\Rightarrow$ (SP):

If $\mathbf{x}$ is limit-affine, then there is a subsequence $\mathbf{u}$ of $\mathbf{x}$ such that $K \lim _{n \rightarrow \infty}\left\|u_{n}-u\right\| \geq \operatorname{diam} \mathbf{u}$ for all $u \in C:=\operatorname{conv} \mathbf{u}, 1<K<N(X)=$ normal structure coefficient (see [10]), contradicting the definition of $N(X)$.

(2) Uniform convexity $\Rightarrow \varepsilon_{0}(X)=\sup \left\{\varepsilon \geq 0 \mid \delta_{X}(\varepsilon)=0\right\}<1 \Rightarrow$ uniformly normal structure:

We have $N(X) \geq\left(1-\delta_{X}(1)\right)^{-1}$ (see [10]) and $\delta_{X}(1)>0 \Leftrightarrow \varepsilon_{0}(X)<1$.

(3) Uniform convexity in every direction (see also [19]) $\Rightarrow$ $\varepsilon_{1}(X):=\sup \left\{\varepsilon \geq 0 \mid\right.$ there exists $u \in X$ with $\|u\| \geq \varepsilon$ and $\left.\delta_{X}(u)=0\right\}<1$ $\Rightarrow(\mathrm{SP})$ :

Here,

$$
\delta_{X}(u):=\inf \left\{1-\frac{1}{2}\|x+y\|\|\| x\|\leq 1,\| y \| \leq 1, x-y=u\right\} .
$$

Let $\mathbf{x}$ be a growing limit-affine sequence. We may assume that $a_{n}=$ $\lim _{m \rightarrow \infty}\left\|x_{m}-x_{n}\right\| \rightarrow a>0$. Choosing $k$ and $m, k<m$, so big that $\left\|x_{k}-x_{m}\right\|-(1-\alpha)\left\|x_{m}-x_{1}\right\| \geq \varepsilon a_{k}, \quad \varepsilon_{1}(X)<\varepsilon<1, \quad 0<\alpha \leq 1$, and $\left(a_{m}-a_{1}\right) \alpha=a_{k}-a_{1}$, and setting $v_{n}=x_{n}-x_{k}, w_{n}=x_{n}-\alpha x_{m}-$ $(1-\alpha) x_{1}$, we obtain $\left\|v_{n}\right\| \rightarrow a_{k},\left\|w_{n}\right\| \rightarrow a_{k},\left\|v_{n}+w_{n}\right\| \rightarrow 2 a_{k}, v_{n}-w_{n}=u$ $=\alpha x_{m}+(1-\alpha) x_{1}-x_{k}$ and $\|u\| \geq \varepsilon a_{k}$ contradicting the definition of $\varepsilon_{1}(X)$.

(4) $k$-uniform rotundity $(k-\mathrm{UR})($ see $[40]) \Rightarrow(\mathrm{SP})$ :

If $X$ is $k-\mathrm{UR}$, then $X$ is (super-)reflexive and there is $\delta>0$ such that:

(I) $\operatorname{det}\left(x_{r}^{*}\left(x_{s}\right), 1\right) \leq 1-\delta$ if $\left\|x_{r}^{*}\right\| \leq 1, \quad r=1, \ldots, k,\left\|x_{s}\right\|-1 \mid \leq \delta$, $s=1, \ldots, \overline{k+1},\left\|x_{k+1}\right\| \geq 1-\delta$.

Here, det is the determinant operation and $\left(\alpha_{r, s}\right):=\left(x_{r}^{*}\left(x_{s}\right), 1\right)$ is the $(k+1) \times(k+1)$-matrix defined by $\alpha_{r, s}=x_{r}^{*}\left(x_{s}\right)$ if $r \leq k$ and $\alpha_{r, s}=1$ otherwise. Using continuity arguments, we find an $\varepsilon>0$ such that (if $\left.\left|\alpha_{r, s}\right| \leq 2\right)$ :

(II) $\operatorname{det}\left(\alpha_{r, s}\right)>1-\delta$ if $\alpha_{k+1, k+1}=1,\left|\alpha_{r, r}-1\right| \leq \varepsilon, r=1, \ldots, k$, $\left|\alpha_{r, s}\right| \leq \varepsilon, r<s \leq k+1$.

If $x_{n} \rightarrow 0$ weakly, $\left\|x_{n}\right\| \rightarrow 1=\lim _{n \rightarrow \infty} \lim _{m \rightarrow \infty}\left\|x_{m}-x_{n}\right\|$ and $\Delta \Sigma_{n} \mathbf{x} \rightarrow$ 0 , then we can pick integers $n_{1}<n_{2}<\cdots<n_{k+1}<n$ and $x_{r}^{*} \in X^{*}$ with $\left\|x_{r}^{*}\right\|=1, r=1, \ldots, k$, such that (II) holds for $\left(\alpha_{r, s}\right)=\left(x_{r}^{*}\left(y_{s}\right), 1\right)$, $y_{s}:=x_{n}-x_{n_{s}}$, and that $\left\|y_{s}\right\|-1 \mid \leq \delta, s=1, \ldots, k+1$, and $\left\|\overline{y_{k+1} \|}\right\| \geq$ $1-\delta$ contradicting (I). 
(5) Nearly uniform convexity (see [25]) $\Rightarrow$ there is a $\delta<1$ such that

(i) $\|x\| \leq \delta$ for some $x \in \operatorname{conv} \mathbf{x}$ if $\left\|x_{n}\right\| \leq 1$ and $\inf _{n \neq m}\left\|x_{n}-x_{m}\right\| \geq \delta$, $\Leftrightarrow X$ is reflexive and there is a $\delta<1$ such that

(ii) $\|x\| \leq \delta$ if $x_{n} \rightarrow x$ weakly, $\left\|x_{n}\right\| \leq 1$ and $\inf _{n \neq m}\left\|x_{n}-x_{m}\right\| \geq \delta$. $\Rightarrow$ (SP):

That (i) implies reflexivity of $X$ is a direct consequence of a characterization of reflexivity of James [26, Theorem 1]. That (i) implies (ii) and that (ii) together with reflexivity implies (i) can be shown using the method of Huff [25].

If $x_{n} \rightarrow 0$ weakly and (iii) $\lim _{m \rightarrow \infty}\left\|x_{m}-x_{n}\right\|=a_{n} \rightarrow 1=$ $\lim _{m \rightarrow \infty}\left\|x_{m}\right\|$, then $\left\|x_{n}-x_{k}\right\|^{-1}\left(x_{n}-x_{k}\right) \rightarrow-a_{k}^{-1} x_{k}$ weakly, and (ii) implies $\left\|x_{k}\right\| \leq \delta a_{k}$ for all sufficiently large $k \in \mathbf{N}$ which contradicts (iii).

(6) $1<\mathrm{BS}(X)=$ bounded sequence coefficient (see $[\mathbf{1 0}]) \Rightarrow(\mathrm{SP})$, or $1<\operatorname{WCS}(X)=$ weakly convergent sequence coefficient (see $[10]) \Rightarrow$ (WSP), resp.:

If $\mathbf{x}$ is limit-affine and $a_{n}:=\lim _{m \rightarrow \infty}\left\|x_{m}-x_{n}\right\|$ converges to some $a>0$, then we can choose a subsequence $\mathbf{u}$ of $\mathbf{x}$ such that $K \lim _{n \rightarrow \infty}\left\|u_{n}-u\right\| \geq a=\lim _{n \rightarrow \infty} \operatorname{diam}\left\{u_{m} \mid m \geq n\right\}$ for all $u \in \operatorname{conv} \mathbf{u}$, $1<K<\operatorname{BS}(X)$ (WCS $(X)$, resp.) which contradicts the definition of $\operatorname{BS}(X)(\mathrm{WCS}(X)$, resp.).

(7) $X$ has a basis with GLD-condition $\Rightarrow$ Bynum's condition [9, Theorem $B] \Rightarrow$ (WSP):

Bynum's proof [9, Theorem B] also works in our situation.

(8) Opial's condition (see [24]) $\Rightarrow$ (WSP):

If $x_{n} \rightarrow 0$ weakly and $\left\|x_{n}\right\| \rightarrow 1$, then Opial's condition implies $\lim _{n \rightarrow \infty}\left\|x_{n}-x_{1}\right\|>1$, if this limit exists, so that $\mathbf{x}$ cannot be a growing limit-affine sequence.

Added in Proof. If $X$ has isonormal structure, then, for every $\varepsilon>0$, there is an equivalent norm |||| $\mid$ on $X$, with respect to which $X$ has normal structure, with $\|x\| \leq\|x\| \leq(1+\varepsilon)\|x\|$ for all $x \in X$. Hence, the class of normally structured spaces isomorphic to $X$ is dense in the class of all spaces isomorphic to $X$ for the Banach-Mazur distance topology. Indeed, choose $\xi=\left(1, \sqrt{\varepsilon^{2}+2 \varepsilon}\|T\|^{-1}\right)$ in the proof of Theorem 4 .

\section{REFERENCES}

[1] J. P. Baillon and R. Schöneberg, Asymptotic normal structure and fixed points of nonexpansive maps, Proc. Amer. Math. Soc., 81 (1981), 257-264.

[2] L. P. Belluce and W. A. Kirk, Fixed point theorems for families of contraction mappings, Pacific J. Math., 18 (1966), 213-217.

[3] _ Nonexpansive mappings and fixed points in Banach spaces, Illinois J. Math., 11 (1967), 474-479. 
[4] L. P. Belluce, W. A. Kirk and E. F. Steiner, Normal structure in Banach spaces, Pacific J. Math., 26 (1968), 433-440.

[5] M. S. Brodskii and D. P. Mil'man, On the center of a convex set, Dokl. Akad. Nauk SSSR, 59 (1948), 837-840 (Russian).

[6] F. E. Browder, Nonlinear mappings of nonexpansive and accretive type in Banach spaces, Bull. Amer. Math. Soc., 73 (1967), 875-881.

[7] _ Semicontractive and semiaccretive nonlinear mappings in Banach spaces, Bull. Amer. Math. Soc.,74 (1968), 660-665.

[8] W. L. Bynum, A class of spaces lacking normal structure, Compositio Math., 25 (1972), 233-236.

[9] _ _ Normal structure of Banach spaces, Manuscripta Math., 11 (1974), 203-209.

[10] _ Normal structure coefficients for Banach spaces, Pacific J. Math., 86 (1980), 427-436.

[11] M. M. Day, Strict convexity and smoothness, Trans. Amer. Math. Soc., 78 (1955), 516-528.

[12] _ Every L-space is isomorphic to a strictly convex space, Proc. Amer. Math. Soc., 8 (1957), 415-417.

[13] M. M. Day, R. C. James and S. Swaminathan, Normed linear spaces that are uniformly convex in every direction, Canad. J. Math., 23 (1971), 1051-1059.

[14] R. DeMarr, Common fixed points for commuting contraction mappings, Pacific J. Math., 13 (1963), 1139-1141.

[15] J. Diestel, Geometry of Banach Spaces-Selected Topics, Springer, Berlin Heidelberg New York (1975).

[16] D. van Dulst and B. Sims, Fixed points of nonexpansive mappings and Chebyshev centers in Banach spaces, preprint.

[17] P. Enflo, Banach spaces which can be given an equivalent uniformly convex norm, Israel J. Math., 13 (1972), 281-288.

[18] B. Fuchssteiner, Iterations and fixpoints, Pacific J. Math., 68 (1977), 73-79.

[19] A. L. Garkavi, On the Čebyšev center of a set in a normed space, Investigations of contemporary problems in the constructive theory of functions, Moscow, (1961), 328-331.

[20] A. A. Gillespie and B. B. Williams, Fixed point theorem for non-expansive mappings on Banach spaces with uniformly normal structure, Appl. Anal., 9 (1979), 121-124.

[21] K. Goebel, An elementary proof of the fixed point theorem of Browder and Kirk, Michigan Math. J., 16 (1969), 381-383.

[22] D. Göhde, Zum Prinzip der kontraktiven Abbildung, Math. Nachr., 30 (1965), 251-258.

[23] J. P. Gossez and E. Lami Dozo, Structure normale et base de Schauder, Bull. de l'Acad. royale de Belgique, (5) 15 (1969), 673-681.

[24] _ Some geometric properties related to the fixed point theory for nonexpansive mappings, Pacific J. Math., 40 (1972), 565-573.

[25] R. Huff, Banach spaces which are nearly uniformly convex, Rocky Mountain J. Math., 10 (1980), 743-749.

[26] R. C. James, Characterizations of reflexivity, Stud. Math., 23 (1964), 205-216.

[27] W. A. Kirk, A fixed point theorem for mappings which do not increase distances, Amer. Math. Monthly, 72 (1965), 1004-1006.

[28] _ Nonexpansive mappings and normal structure in Banach spaces, Proc. Conf. on Geom. of Banach spaces, Univ. of Iowa, (July 1981).

[29] _ Nonexpansive mappings in metric and Banach spaces, Rend. Seminario Mat. e Fis. Milano, (to appear).

[30] T. Landes, Das Iterationstheorem und Fixpunktsätze bei normaler Struktur, Dissertation, Paderborn 1981. 
[31] , A characterization of totally normal structure, Arch. Math., 37 (1981), $248-255$.

[32] T. C. Lim, A fixed point theorem for families of nonexpansive mappings, Pacific J. Math., 53 (1974), 487-493.

[33] - Characterizations of normal structure, Proc. Amer. Math. Soc., 43 (1974), 313-319.

[34] E. Maluta, Characterization of normal type structure by means of sequences, Ist. Lombardo Accad. Sci. Lett. Rend. A 113 (1979), 436-441.

[35] J. R. Partington, Equivalent norms on spaces of bounded fuctions, Israel J. Math., 35 (1980), 205-209.

[36] Subspaces of certain Banach sequence spaces, Bull. London Math. Soc., 13 (1981), 162-166.

[37] J. Rainwater, Local uniform convexity of Day's norm on $c_{0}(\Gamma)$. Proc. Amer. Math. Soc., 22 (1969), 335-339.

[38] R. Schöneberg, Fixpunktsätze für einige Klassen kontraktionsartiger Abbildungen in Banachräumen über einen Fixpunktindex, eine Zentrummethode und die Fixpunkttheorie nichtexpansiver Abbildungen, Dissertation, Aachen 1977.

[39] P. Soardi, Schauder bases and fixed points of nonexpansive mappings, Pacific J. Math., (to appear).

[40] F. Sullivan, $A$ generalization of uniformly rotund Banach spaces, Canad. J. Math., 31 (1979), 628-636.

[41] V. Zizler, On some rotundity and smoothness properties of Banach spaces, Diss. Math. Rozprawy Mat., 87 (1971), 1-37.

Received February 8, 1982 and in revised from March 10, 1982.

UNIVERSity OF PADERBoRN

WARBURGERSTR. 100

D-4790 PADERBORN

WEST GERMANY 



\section{PACIFIC JOURNAL OF MATHEMATICS \\ EDITORS}

DONALD BABBITT (Managing Editor)

University of California

Los Angeles, CA 90024

Hugo RossI

University of Utah

Salt Lake City, UT 84112

C. C. MOOre and ARThur OGus

University of California

Berkeley, CA 94720
J. Dugundi

Department of Mathematics

University of Southern California

Los Angeles, CA 90089-1113

R. FINN and H. SAMELSON

Stanford University

Stanford, CA 94305

ASSOCIATE EDITORS

$\begin{array}{lllll}\text { R. Arens } & \text { E. F. Beckenbach } & \text { B. H. Neumann } & \text { F. Wolf } & \text { K. Yoshida }\end{array}$ $(1906-1982)$

\section{SUPPORTING INSTITUTIONS}

UNIVERSITY OF ARIZONA

UNIVERSITY OF BRITISH COLUMBIA

CALIFORNIA INSTITUTE OF TECHNOLOGY

UNIVERSITY OF CALIFORNIA

MONTANA STATE UNIVERSITY

UNIVERSITY OF NEVADA, RENO

NEW MEXICO STATE UNIVERSITY

OREGON STATE UNIVERSITY
UNIVERSITY OF OREGON

UNIVERSITY OF SOUTHERN CALIFORNIA

STANFORD UNIVERSITY

UNIVERSITY OF HAWAII

UNIVERSITY OF TOKYO

UNIVERSITY OF UTAH

WASHINGTON STATE UNIVERSITY

UNIVERSITY OF WASHINGTON 


\section{Pacific Journal of Mathematics}

\section{Vol. 110, No. $1 \quad$ September, 1984}

Wojciech Abramczuk, A class of surjective convolution operators $\ldots \ldots \ldots \ldots 1$

K. Adachi, Extending bounded holomorphic functions from certain

subvarieties of a weakly pseudoconvex domain $\ldots \ldots \ldots \ldots \ldots \ldots$

Malvina Florica Baica, An algorithm in a complex field and its application

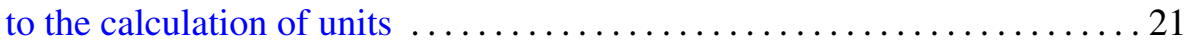

Giuliana Bianchi and Robert Cori, Colorings of hypermaps and a

conjecture of Brenner and Lyndon $\ldots \ldots \ldots \ldots \ldots \ldots \ldots \ldots \ldots \ldots \ldots \ldots$

Ronald James Evans, Determinations of Jacobsthal sums . . .......... 49

Leslie Foged, Characterizations of $\aleph$-spaces .................... 59

Nassif A. Ghoussoub and Paulette Saab, Weak compactness in spaces of

Bochner integrable functions and the Radon-Nikodým property . . . . . . 65

J. Gómez Gil, On local convexity of bounded weak topologies on Banach

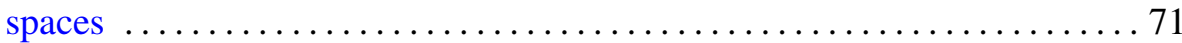

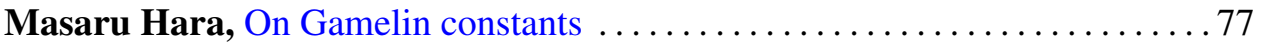

Wilfried Hauenschild, Eberhard Kaniuth and Ajay Kumar, Harmonic

analysis on central hypergroups and induced representations $\ldots \ldots \ldots 83$

Eugenio Hernandez, An interpolation theorem for analytic families of

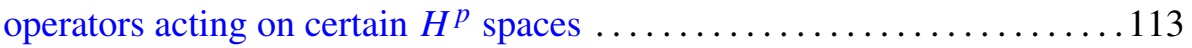

Thomas Alan Keagy, On "Tauberian theorems via block-dominated

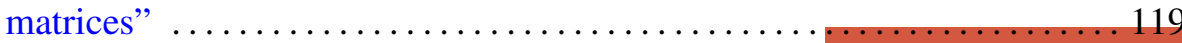

Thomas Landes, Permanence properties of normal structure $\ldots \ldots \ldots \ldots \ldots 125$

Daniel Henry Luecking, Closed ranged restriction operators on weighted

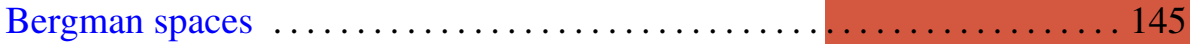

Albert Thomas Lundell, The $p$-equivalence of $\mathrm{SO}(2 n+1)$ and $\mathrm{Sp}(n) \ldots \ldots 161$

Mark D. Meyerson, Remarks on Fenn's "the table theorem" and Zaks' "the chair theorem" ..................................... 167

Marvin Victor Mielke, Homotopically trivial toposes . . . . . . . . . . 171

Gerard J. Murphy, Hyperinvariant subspaces and the topology on Lat A . . 183

Subhashis Nag, On the holomorphy of maps from a complex to a real manifold

Edgar Milan Palmer and Robert William Robinson, Enumeration of self-dual configurations ................................. 203

John J. Walsh and David Clifford Wilson, Continuous decompositions

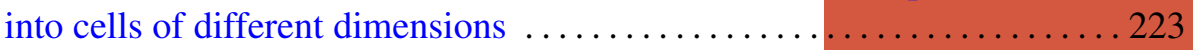

Walter John Whiteley, Infinitesimal motions of a bipartite framework .....233 